Case Report

\title{
A Rare Malignant Transformation of an Ovarian Cystic Teratoma: A Case Report
}

\author{
Manju Rachel Mathew (D, ${ }^{1}$ Anita Ramdas, ${ }^{1}$ Susy S. Kurian, ${ }^{1}$ \\ Linu Kuruvilla $\mathbb{}^{1},{ }^{2}$ and Neelima Singh ${ }^{3}$ \\ ${ }^{1}$ Department of Pathology, Pondicherry Institute of Medical Sciences, Ganapathichettikulam, Kalapet, Puducherry 605014, India \\ ${ }^{2}$ Department of Radiodiagnosis, Pondicherry Institute of Medical Sciences, Ganapathichettikulam, Kalapet, Puducherry 605014, India \\ ${ }^{3}$ Department of Obstetrics and Gynecology, Pondicherry Institute of Medical Sciences, Ganapathichettikulam, Kalapet, \\ Puducherry 605014, India
}

Correspondence should be addressed to Manju Rachel Mathew; manjumathew36@hotmail.com

Received 8 March 2018; Accepted 14 June 2018; Published 9 July 2018

Academic Editor: Stefan Pambuccian

Copyright (C) 2018 Manju Rachel Mathew et al. This is an open access article distributed under the Creative Commons Attribution License, which permits unrestricted use, distribution, and reproduction in any medium, provided the original work is properly cited.

Mature cystic teratoma (MCT) is the commonest germ cell neoplasm of the ovary but malignant transformation is a rare occurrence (1-2\%). Of these malignancies documented in literature the commonest are squamous cell carcinoma and adenocarcinoma. Urothelial carcinomas arising in an MCT are a rare occurrence and only 7 cases have been reported in literature. We report a case of an MCT which was complicated by the presence of urothelial carcinoma confirmed on histopathological examination.

\section{Introduction}

According to the 2014 WHO classification, mature cystic teratomas (MCT) constitute approximately $20 \%$ of all ovarian neoplasms also being the commonest germ cell tumor [1]. They are tumors which can form mature tissues derived from ectoderm, mesoderm, and endoderm and are usually cystic [2]. The majority of these tumors are benign with malignant transformation being rare and reported in only $1-2 \%$ of cases $[3,4]$. Among the malignant transformations, squamous cell carcinoma and adenocarcinoma are the most commonly reported [5]. Urothelial carcinomas arising in an MCT is rare [3-9]. This is a report of such a case in a 50-yearold lady.

\section{Case Report}

A 50-year-old lady presented to the Pondicherry Institute of Medical Sciences Hospital at Puducherry, India, with a complaint of acute abdominal pain. Contrast enhanced computed tomography (CECT) demonstrated the presence of bilateral ovarian mature cystic teratomas. Contrast enhancement within the right ovarian cyst suggested the possibility of malignant transformation (Figure 1). Tumor marker carbohydrate antigen- (CA-) 125 was $27 \mathrm{IU} / \mathrm{mL}$ (normal $<35 \mathrm{IU} / \mathrm{mL}$ ). She underwent total abdominal hysterectomy and bilateral salpingo-oophorectomy.

Gross examination showed the right ovary to be cystic and measured $12 \mathrm{cms}$ in diameter and is predominantly smooth except for an area of $3 \mathrm{~cm}^{2}$ which had blunt pale soft projections (Figure 2). The left ovary was grossly normal measuring $3 \mathrm{cms}$ in greatest diameter.

Microscopically the left and right ovary showed various mature tissues including bronchial mucosa, apocrine glands, cartilage, and skin with adnexal structures. The microscopy of the soft pale projections of the right ovary had papillary structures with fibrovascular cores which were lined by transitional epithelium exhibiting nuclear pleomorphism, hyperchromatism, and increased mitotic activity (Figure 3 ). There was evidence of invasion of the ovarian stroma by nests of malignant epithelial cells (Figure 4). The inked ovarian capsular surface was free of tumor. Immunohistochemistry 


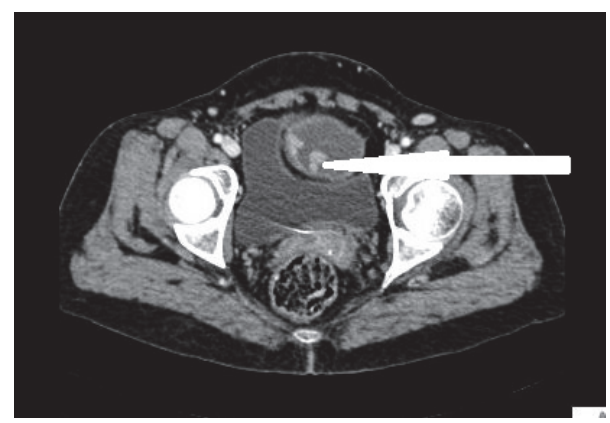

Figure 1: Contrast enhanced computed tomography (CE-CT) image showing cystic right ovary with arrow pointing to enhanced solid densities within the cyst.

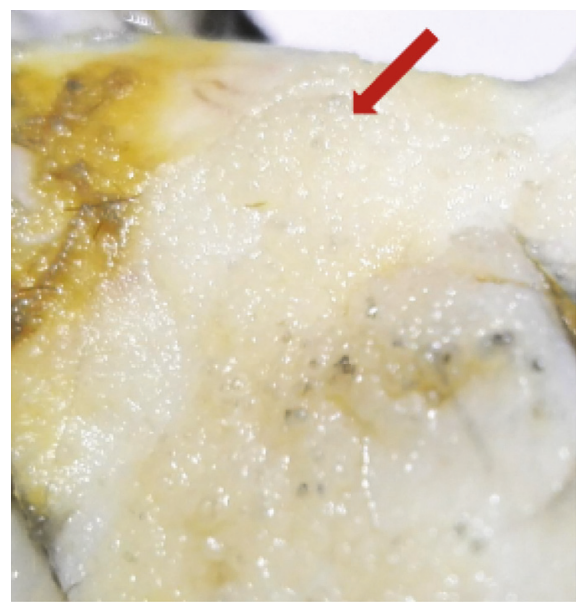

FIGURE 2: Blunt pale projections on an otherwise smooth cyst wall.

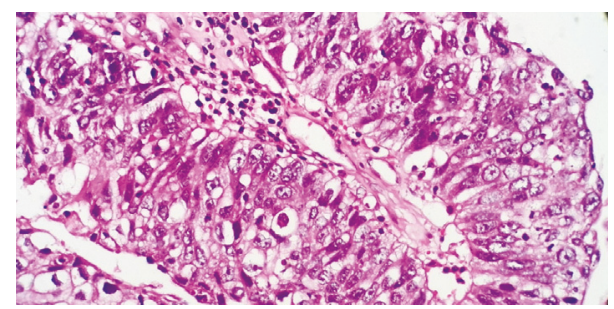

FIGURE 3: High power view of histomorphology of urothelial carcinoma (H\&E 400X magnification).

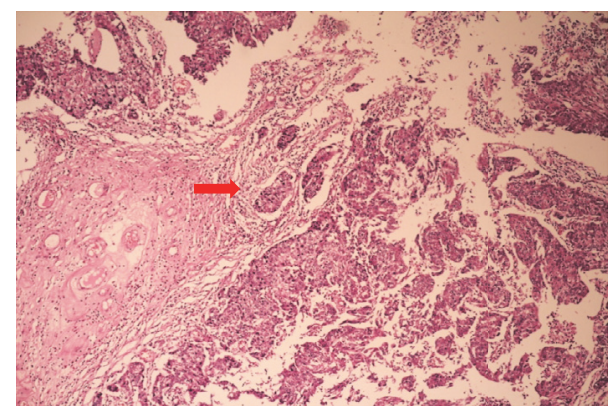

FIGURE 4: Low power view of urothelial carcinoma with arrow pointing at nests of invasive malignant epithelial cells in ovarian stroma (H\&E 10X magnification).

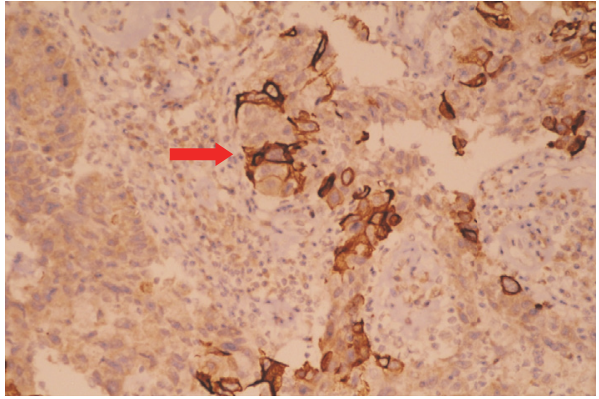

FIGURE 5: High power view of tumor cells showing cytoplasmic and membranous positivity for Uroplakin II (IHC 40X magnification).

(IHC) of the urothelial carcinoma showed cytoplasmic and membrane positivity for Uroplakin II (Figure 5). A diagnosis of ovarian cystic teratoma with primary invasive urothelial carcinoma (TNM stage pTlaNxMx) was made based on the Pathological Stage Classification by the American Joint Committee on Cancer (AJCC) $8^{\text {th }}$ edition [10].

The patient was reviewed till 3 months following surgery and follow-up CECT revealed no evidence of recurrent tumor in the abdomen and pelvis.

\section{Discussion}

Urothelial carcinomas complicating an MCT is rare and only 7 such cases have been reported in literature so far [3-9]. The diagnosis of primary invasive urothelial carcinoma in the present case was made by morphology and immunohistochemically demonstrating Uroplakin II in the malignant cells.

A review of the salient features of the 7 prior cases and that of the present patient is presented in Table 1 [3-9].

Studies by Al-Rayyan et al. and Kikkawa et al. showed varying number of patients with malignant transformation of an ovarian MCT who were postmenopausal or premenopausal thus indicating that menopause itself does not pose as a risk factor for malignant transformation in an MCT $[4,11]$. Older age group ( $>45$ years), elevated levels of CA-125 $(35 \mathrm{IU} / \mathrm{mL})$, preoperative tumor size $(>9.9 \mathrm{~cm})$, and characteristic imaging findings were noted as independent risk factors for malignant transformation of an MCT $[3,11$, 12]. The present case differed in that no elevation of CA-125 $(27 \mathrm{IU} / \mathrm{mL}$ ) was noted unlike majority of the previous cases [3-9].

The pathogenesis of urothelial carcinoma in an MCT was hypothesized in two studies from Korea which noted the presence of normal urothelium adjacent to the urothelial carcinoma hence suggesting that prolonged and persistent irritation of the urothelium by lipid material induced the secondary carcinomatous change $[7,9]$.

Lee et al and Dasgupta et al. stated the importance of differentiating the urothelial carcinoma arising in an MCT from a primary transitional cell carcinoma of the ovary (TCC-O) which is a subtype of surface epithelial tumors $[6,7]$. Lee stated that the urothelial carcinoma of the urinary tract expressed Uroplakin III but is negative for WT1. On the contrary TCC-O is negative for Uroplakin III and positive 


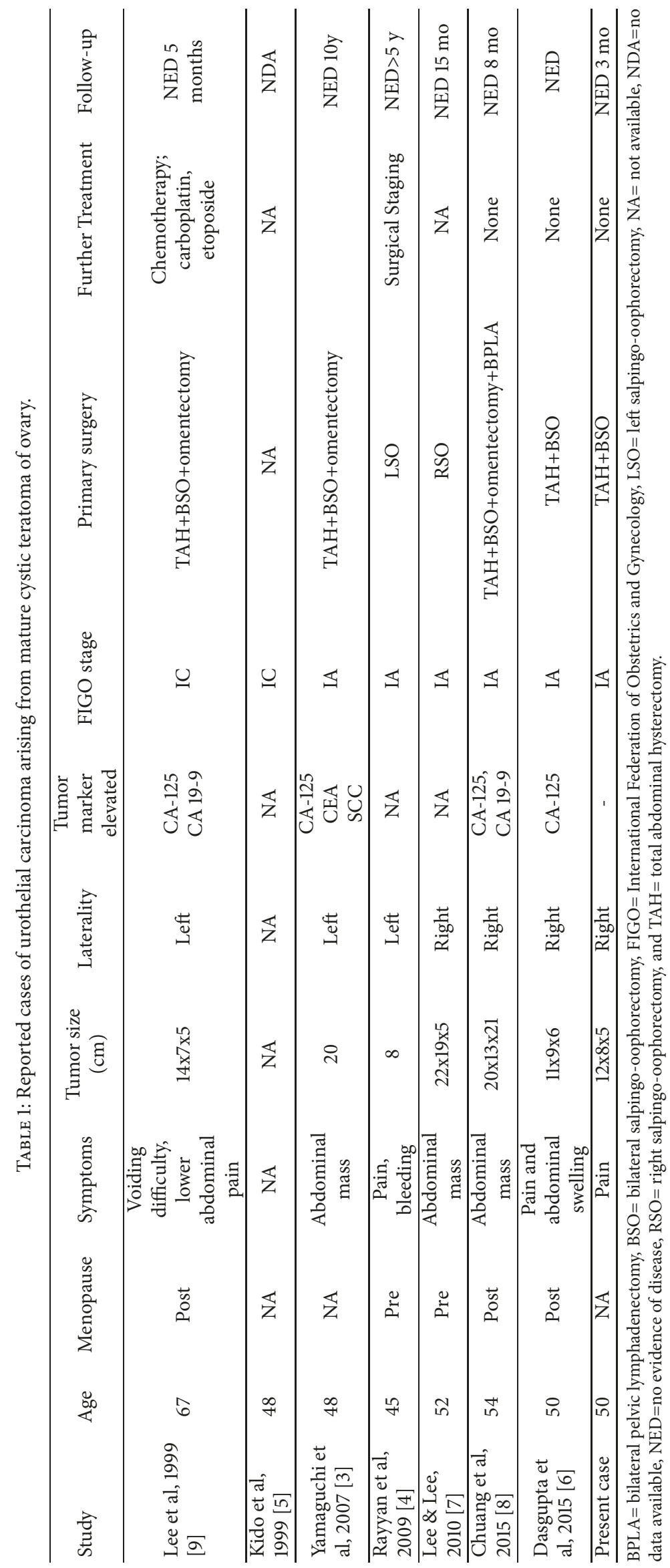


for WT1 [7]. Smith et al. and Li et al. in 2014 showed that Uroplakin II is a more sensitive immunohistochemical marker than Uroplakin III for detecting urothelial carcinoma of the urinary tract $[13,14]$. In the present case the urothelial carcinoma arising in the MCT showed cytoplasmic and membrane positive staining for Uroplakin II suggesting its origin from the urothelium of the teratoma. The possibility of metastatic deposits from a primary urinary tract carcinoma was ruled out by radiological evaluation showing no lesion in the urinary system. Hence a diagnosis of primary invasive urothelial carcinoma arising in a mature cystic teratoma of the ovary was made.

\section{Conclusion}

Malignant transformation in an MCT is a rare complication but must be suspected in high risk cases of age $>45$ years, size larger than $9 \mathrm{~cm}$, and elevated tumor marker CA-125 levels. Urothelial carcinomas arising in an MCT of ovary is a rare occurrence and to the best of our knowledge this is the eighth such case reported in literature.

\section{Data Availability}

The authors declare that the data cited in this report are available in the references mentioned in this paper and can be accessed from Pubmed.

\section{Conflicts of Interest}

The authors declare that they have no conflicts of interest regarding the publication of this paper.

\section{References}

[1] R. J. Kurman, M. L. Carcangiu, C. S. Herrington, and R. H. Young, Eds., WHO Classification of Tumours of Female Reproductive Organs, IARC, Lyon, 2014.

[2] Rosai J and L. V. Ackerman, "Ovary," in Rosai and Ackermans Surgical Pathology, pp. 1553-1635, Elsevier, New Delhi, India, 10th edition, 2011.

[3] K. Yamaguchi, M. Mandai, K. Fukuhara et al., "Malignant transformation of mature cystic teratoma of the ovary including three cases occurring during follow-up period," Oncology Reports, vol. 19, no. 3, pp. 705-711, 2008.

[4] E. S. Al-Rayyan, W. J. Duqoum, M. S. Sawalha et al., "Secondary malignancies in ovarian dermoid cyst," Saudi Medical Journal, vol. 30, no. 4, pp. 524-528, 2009.

[5] A. Kido, K. Togashi, I. Konishi et al., "Dermoid cysts of the ovary with malignant transformation: MR appearance," American Journal of Roentgenology, vol. 172, no. 2, pp. 445-449, 1999.

[6] S. Dasgupta, D. Bose, N. Bhattacharyya, and P. Biswas, "Mature cystic teratoma with malignant transformation of teratomatous urothelial cells: Rare case presentation," Clinical Cancer Investigation Journal, vol. 4, no. 4, p. 534, 2015.

[7] O.-J. Lee and H.-C. Lee, "Urothelial (Transitional Cell) carcinoma arising in mature cystic teratoma: A case report," The Korean Journal of Pathology, vol. 44, no. 6, pp. 666-669, 2010.
[8] H.-Y. Chuang, Y.-T. Chen, T.-L. Mac et al., "Urothelial carcinoma arising from an ovarian mature cystic teratoma," Taiwanese Journal of Obstetrics and Gynecology, vol. 54, no. 4, pp. 442-444, 2015.

[9] H. H. Lee, J. Y. Shim, and C. Lee, "A case of papillary transitional cell carcinoma arising from the benign cystic teratoma of ovary," Korean Journal of Obstetrics \& Gynecology, vol. 42, pp. 11231126, 1999.

[10] S. Movahedi-Lankarani, "Protocol for the examination of specimens from patients with primary tumors of the ovary, fallopian tube or peritoneum," College of American Pathologists, 2017.

[11] F. Kikkawa, A. Nawa, K. Tamakoshi et al., "Diagnosis of squamous cell carcinoma arising from mature cystic teratoma of the ovary," Cancer, vol. 82, no. 11, pp. 2249-2255, 1998.

[12] G. W. H. Stamp and E. M. McConnell, "Malignancy arising in cystic ovarian teratomas," BJOG: An International Journal of Obstetrics \& Gynaecology, vol. 90, no. 7, pp. 671-675, 1983.

[13] S. C. Smith, S. K. Mohanty, L. P. Kunju et al., "Uroplakin II outperforms uroplakin III in diagnostically challenging settings," Histopathology, vol. 65, no. 1, pp. 132-138, 2014.

[14] W. Li, Y. Liang, M. T. Deavers et al., "Uroplakin II is a more sensitive immunohistochemical marker than uroplakin III in urothelial carcinoma and its variants," American Journal of Clinical Pathology, vol. 142, no. 6, pp. 864-871, 2014. 


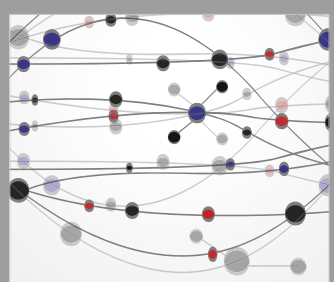

The Scientific World Journal
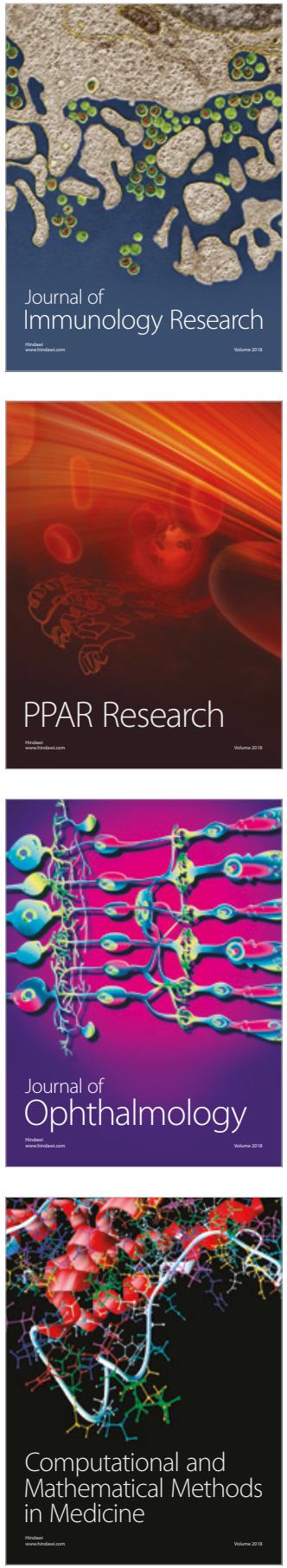

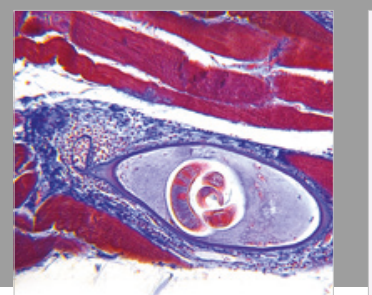

Gastroenterology Research and Practice

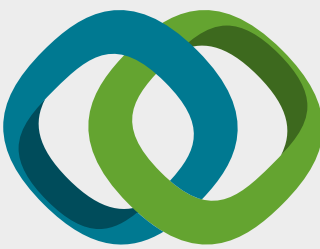

\section{Hindawi}

Submit your manuscripts at

www.hindawi.com
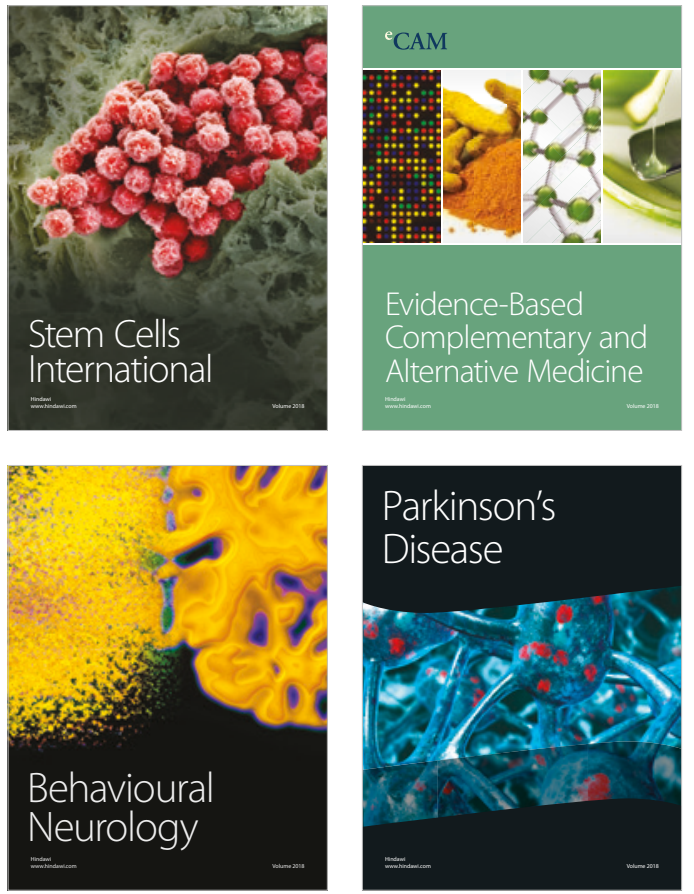

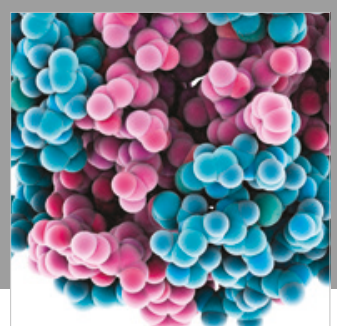

ournal of

Diabetes Research

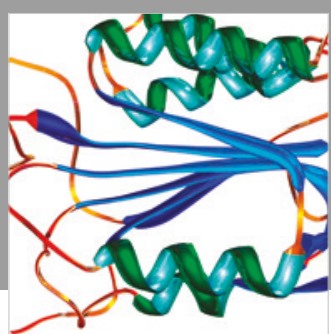

Disease Markers
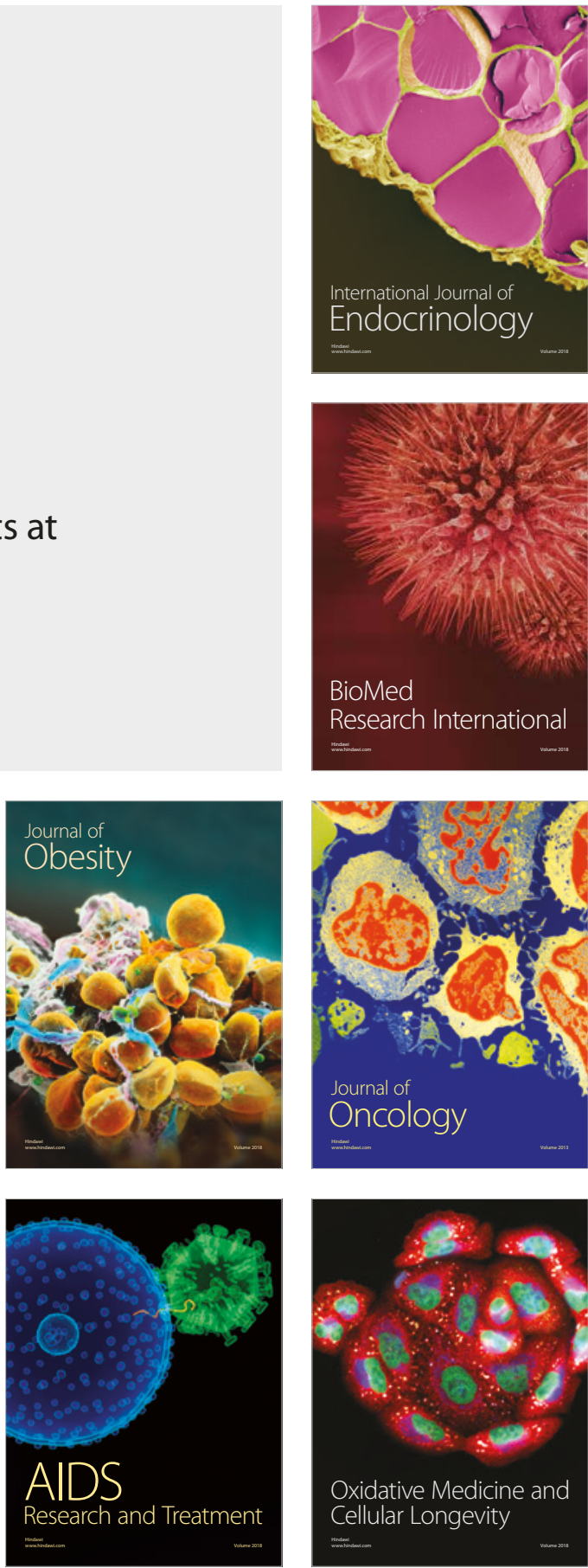